\title{
Knowledge Organization and Representation in Digital Environments: Relations Between Ontology and Knowledge Organization ${ }^{\dagger}$
}

\author{
Carlos Henrique Marcondes \\ Fluminense Federal University, Department of Information Science, R. Lara Vilela, 126, \\ 24210-590, Niterói, Rio de Janeiro, Brazil, phone 5521 26299758, <marcon@vm.uff.br>
}

\begin{abstract}
Carlos Henrique Marcondes holds a $\mathrm{PhD}$ in information science and is associate professor at Fluminense Federal University, Department of Information Science, Niterói, Rio de Janeiro, Brazil. His research interests include knowledge organization and representation in digital environments, use of semantic web technologies, information retrieval, and foundational issues.
\end{abstract}

Marcondes, Carlos Henrique. Knowledge Organization and Representation in Digital Environments: Relations Between Ontology and Knowledge Organization. Knowledge Organization. 40(2), 115-122. 47 references.

\begin{abstract}
Knowledge organization faces the challenge of contributing to the management of the amount of knowledge produced and available in the Web environment. Computational ontologies are new artifacts for knowledge recording and processing and also one of the foundations of the semantic web; they pose new challenges to knowledge organization in clarifying its interdisciplinary relations and specific role within knowledge management disciplines. What are its relations to ontology? A draft of these relations is presented, obtained from authors who discuss foundational issues, with the aim of identifying the actual role of knowledge organization in the Web environment. While ontology discusses the ultimate nature of being, knowledge organization emphasizes additional practical issues unfolding all possible manifestations of that which is. A primary question is: how to seek information, how to be informed?
\end{abstract}

Received 15-12-2012; Revised 13-1-2013; Accepted 16-1-2013

† This paper is a revised and extended version of a previous one presented in the Brazilian ISKO 2011 Conference

\subsection{Introduction}

General rules that are always applicable for the choice of names of subjects can no more be given than rules without exception in grammar. Usage in both cases is the supreme arbiter- the usage, in the present case, not of the cataloger, but of the public in speaking of subjects (Cutter 1904, 69). Nowadays, knowledge organization (KO) faces the challenge of contributing to the management of growing stocks of knowledge records produced and available on the Web in order to enable their reuse and appropriation, as required by the emergence of the information society. The Web poses new challenges to KO. A comprehensive proposal aimed at organizing Web con- tent in an innovative mode, different from current computational processing paradigm is the Semantic Web proposal. The Semantic Web will bring structure to the meaningful content of Web pages, creating an environment where software agents roaming from page to page can readily carry out sophisticated tasks for users. (Berners-Lee, Hendler, and Lassila 2001, 2).

The importance of the Semantic Web vision to $\mathrm{KO}$ is emphasized by Hjörland (2007, 371): "and of course in particular the new concept considered by many the most important frontier in knowledge organization: 'the semantic web." The Semantic Web proposal is ambitious with respect to KO. Berners-Lee, Hendler, and Lassila (2001, 2) write: 
The Semantic Web is not "merely" the tool for conditioning individual tasks that we have discussed so far. In addition, if properly designed, The Semantic Web can assist the evolution of human knowledge as a whole.

Based on the vision of the Semantic Web, different theoretical and practical proposals have posed the question of how to assign semantics to Web content to allow programs to process it more efficiently and thus help us with the enormous task of organizing and provide access to it. Within this context, ontologies are new computational artifacts that can provide computational semantics to web content, allowing programs, in addition to data processing, make inferences about this content. Different communities have developed computational ontologies with varying degrees of success. However, with the exception of some cases, we are far from the vision proposed by the Semantic Web (Berners-Lee, Hendler, and Lassila 2001).

Research in ontology has an interdisciplinary nature, involving contributions from philosophy, logic, computer science, among other disciplines, and, increasingly, contributions from $\mathrm{KO}$, too, aimed at successfully facing the trends previously posed. The relationship between these disciplines and the KO legacy due to the development of bibliographic classifications is recognized in the current ontology literature (Smith and Welty 2001). Several authors in information science (Wersig and Neveling 1975; Saracevic 1995) have stressed its interdisciplinary relationships along the history of the discipline in an attempt to clarify its own scope.

Today, the emergence of the Semantic Web proposal and of ontologies as new artifacts to record humanity's knowledge pose new challenges to the comprehension of $\mathrm{KO}$ relationships and their roles within the different cognitive and knowledge management sciences. What should the relationships between disciplines of ontology and $\mathrm{KO}$ be? How important is ontology to $\mathrm{KO}$ and vice-versa? As Epistemology discusses the problem of how we know and ontology discusses and formally describes the things we know, what does KO discusses? What is its role among the spectrum of disciplines engaged with the problem of large scale knowledge management and appropriation, especially in today's Web environment? There is a need to clarify the nature of the relations with those disciplines in order to develop fruitful relations with them. How can such artifacts, considered as bases of the Semantic Web, be useful to KO? What may be the contribution of ontology to KO? What may be the specific contribution of $\mathrm{KO}$ to the development of computational ontologies? To what extent are both disciplines complementary?

This work develops an analysis and sketches the relationships between KO, with an emphasis on Ranganathan's
Faceted Classification Theory, and disciplines such as formal and computational ontology. Texts by authors with a focus on the foundations of these disciplines such as Guarino, Smith, and Guizzardi are analysed. Authors' claims related to foundational questions are contrasted as to the discipline objectives, the need, and the grade of formalization, the rules for building taxonomies, the proposal of high level/foundational ontologies, and the assortment of properties. The aim is to identify the $\mathrm{KO}$ specific role within the disciplines which deal with the management of humanity's stock of knowledge, mainly in digital environments inspired by the Semantic Web proposal. The work is organized as follows. After this introduction, Section 2 discusses the aims of formal ontology, and Section 3 the aims of KO. Section 4 presents a discussion of possible methodological intersections and complementary approaches between the two disciplines. Finally, Section 5 presents conclusions.

\subsection{What are the aims of ontology?}

For some time, issues related to the modeling of specific domains in computer environments have required inquiries on the ultimate nature of the entities in these domains. This is a requirement to ensure correspondence between these models and reality, thus enabling computers to reason correctly on these models. To cope with these issues, contributions are needed from formal ontology. The discipline applies principles of philosophical ontology in the sense of Husserl's Logical Investigations to the modelling of computer systems. Authors such as Guarino $(1997,1)$ define formal ontology as dealing with "formal distinctions between the elements of a domain, independently of their actual reality;" Guarino $(1995,5)$ claims that it deals with a priori distinctions:

- among the entities of the world (physical objects, events, regions, quantities of matter;

- among the meta-level categories used to model the world (concepts, properties, qualities, states, roles, parts.

Discussing the scope of formal ontology, Guarino (1995, 2) claims that "As such, formal ontology is a recent expression of traditional ontology, intended as the branch of philosophy which deals with the a priori nature of reality," i.e., a nature which is always present in reality, independent of the domain considered. Stressing this particular aspect of domain independence, Guarino and Guizzardi $(2006,117)$ claim that: "in particular, so-called formal ontology is completely neutral for what concerns its domain of application." 
Formal ontology claims itself to be an autonomous knowledge discipline having relationships with computer science especially with artificial intelligence, information science, logic, and philosophical ontology. Relevant contributions have been made by researchers around forums such as IAOA (The International Association for Ontology and its Applications), events such as FOIS (International Conference on Formal Ontology in Information Systems) - which is in its $7^{\text {th }}$ edition in 2012 - and the Journal of Applied Ontology.

From a historical perspective, disciplines such as artificial intelligence have been changing their focus as Guarino (1995, 625) stresses:

AI researchers seem to have been much more interested in the nature of reasoning rather than in the nature of the real world. Recently, however, the potential value of task-independent knowledge bases (or 'ontologies') suitable to large-scale integration has been underlined in many ways.

According to the author, the rise of computational ontology, although it has roots in AI, shows a sharp difference between task-independent knowledge bases or ontologies, and reasoning processes, which were the focus of early AI. That seems a fundamental change in perspective as it poses the question of how to develop a representation of (or to model, according to Le Moigne 1990, a specific domain which is independent of any particular computational application).

Barry Smith and Christopher Welty $(2001,4)$ claim that: "Philosophical ontology is the science of what is, of the kinds and structures of objects, properties, events, processes and relations in every area of reality"; in addition, Smith $(2006,2)$ claims that:

It seeks not explanation but rather a description of reality in terms of a classification of entities that is exhaustive in the sense that it can serve as an answer to such questions as: What classes of entities are needed for a complete description and explanation of all the goings-on in the universe? Or: What classes of entities are needed to give an account of what makes true all truths? Or: What classes of entities are needed to facilitate the making of predictions about the future?

Smith $(2006,5)$ makes a significant distinction between philosophical ontology and science as he says that:

Philosophical ontology is a descriptive enterprise. It is distinguished from the special sciences not only in its radical generality but also in its primary goal or focus: it seeks, not predication or explanation, but rather taxonomy.

McGuinness (2003) defines a computational ontology as an artifact where there are formally declared controlled vocabularies, relations between classes, hierarchical classsubclass relations, properties of classes, value restrictions at the class level, inclusion of individuals to classes, disjointedness between classes, arbitrary logical relations between terms, and inverse and part-whole relations. As stated before (Smith 2006), the backbone of every ontology is a taxonomic structure, in other words, classsubclass and class-instance relations. Thus, according to those authors, foundational issues related to the application of ontology to modeling systems and knowledge representation in digital environments are:

- What exists?

- Which are the entities that exist in a specific domain?

- How are they?

- What are their differences?

- What are their similarities?

- Which entity is similar to which entity?

- What are their properties?

Ontological analysis aims at, as stated by Guarino and Welty $(2009$, 8), identifying all logical consequences of the choices made when modelling a domain, and enabling inferences which are logically and ontologically correct. Some important methodological contributions of ontological analysis to domain modelling are:

- identifying metaproperties of the properties occurring in a domain, such as those which ensure Identity, Dependence, and Integrity criteria to individuals involved in those properties (Guarino 1997);

- identifying and analyzing roles as features which can add semantics and precision to modelling (Masolo et al. 2004); and,

- identifying types of formal-ontological relations (Smith and Grenon 2004).

According to different authors (Guarino 1998, Guizzardi 2005), formal ontology should provide the bases to the development of the so-called application ontologies, computational artifacts (Guarino 1998) that model a specific application and are tied to computational systems which this author calls "ontology-driven systems." Guarino (1998) proposes that ontologies may be developed in different levels of generality: top-level or foundational ontologies that provide very general concepts like space, time, matter, object, event, action, etc., which are independent of a particular domain; domain ontologies and 
task ontologies of specialized concepts provided by toplevel ontologies providing concepts to a generic domain or task like medicine and diagnosis; and application ontologies that provide concepts which are specializations of the former to a particular application.

The efficiency of such systems depends on the accuracy of these application ontologies. The importance of accurate knowledge representations in digital environments is stressed by Davis, Shrobe, and Szolovits (1993, 19): "Imperfect surrogates mean incorrect inferences .... If the world model is somehow wrong (and it must be) some conclusions will be incorrect, no matter how carefully drawn." All of these proposals constitute a comprehensive methodological framework for the development of domain systems.

\subsection{What are the aims of knowledge organization?}

Most past classification schemas are just evolutionary proposals of previous ones. The informal natures of past precoodinated classification schemas are emphasized by Vickery (2008b):

In an enumerative, precoordinated classification, the hierarchical links ostensibly represent the generic relation between a class and its subclasses, but in practice they may also be used for the class-membership relation. The nature of the link becomes somewhat indeterminate when, for example, a part or attribute is shown as a subclass of an entity.

Consequently, KOS operations depended on human intervention demanding implicit knowledge. The lack of principles and formalism in such systems needed to be complemented with the expertise and experience of information professionals when faced with concrete problems of classification and seeking books in a library cata$\log$. In such scenarios, there was a clear need of methodologies and principles to build classification schemas.

This situation led S. R. Ranganathan to write his Prolegomena to Library Classification. What Ranganathan proposes in the Prolegomena is not a classification system in itself but principles for the development of such systems. According to Ranganathan $(1967,30)$ : “As a result classification was taught in about 30 schools of these countries, on the basis of Postulates and Guiding Principles." The principles proposed are organized throughout the Prolegomena in Normative Principles or Laws, Canons, and Postulates.

When exposing the Analytico-Synthetic Classification Theory, Ranganathan (1967) poses two requisites: 1) to propose classification systems which could cope with the dynamism of contemporary knowledge, an issue which had always been a preoccupation of previous classification systems, although systems such as Dewey and even UDC are both flexible enough to cope with the emergence of new disciplines or subjects; indeed, Foskett (1996) proposes hospitality as a quality criteria for classification systems.

At the same time, the Analytico-Synthetic Classification must 2) account for representing compound subjects as they appear in knowledge records-books, documents, scientific articles-in contrast to the previous system, which Ranganathan calls "enumerative" due to the fact that they simply enumerate current knowledge and define a unique, rigid position for a document within it. The ability to cope with compound subjects reveals a central issue both in theory and practice of KOS, which is a preoccupation with users. Actually, one difference between faceted classification systems and previous ones is their focus on user needs and retrieval.

$D D C$ and LCC belong to the type of system that was designed for shelving purposes, while UDC, from the beginning, was developed as a bibliographic system designed for retrieval purposes and consequently belong to the same-more advanced-type of system as the $\mathrm{BC}$ system (Bliss Bibliographic Classification) and the CC (Colon Classification) (Ranganathan 1968).

When developing information systems, information science always considered users' information needs (Buckland 1991). Modern KO theories always consider users' points of view when developing KOS, and domain analysis has been proposed as a solid base to the development of KOS (Hjörland and Albrechtsen 1995). Domain analysis is an approach strongly driven by users needs. The concept of relevance, so important to information science (Saracevic 1975), was always considered as a parameter in the Ranganathan formulations: "the issue of relevance appears frequently in the theories of both Ranganathan and the CRG" (Spiteri 1998, 5). This is what Ranganathan (1967) aimed when he proposes the Canon of Relevance, the Canon of Relevant Sequence, and the Canon of Helpful Sequence. The concept of relevance poses the question: for whom is the classification scheme is being developed?

The third of the Laws from Ranganathan states for every reader his book. Attending to user needs related to the recovery of compound subjects thus enforces the requisites he recommends for a classification scheme. Ranganathan $(1967,88)$ developed the concept of facet, defined as "any component-be it a basic subject or an isolate_of a compound subject." When developing specific KOS to a domain, categories of the basic taxonomic structure are deployed according to PMEST categoriesPersonality, Matter, Energy, Space, and Time-being an expression of these facets in a domain; the objective is to 
represent a domain with its same characteristics - their facets-that will enable their combination to represent compoud subjects. Facets thus enable users to access diferent expressions of this entity, as they are discussed in knowledge records-books, documents. Facet analysis aims at making different possible access points explicit, that is, it makes explicit the links between a classification system and the knowledge records to which it serves as a finding aid.

Thus facet analysis aims to answer the question: under what aspect does that which exists in the world manifest? To hit this objective, Ranganathan proposes an analysis which makes a division characteristic explicit to the next level of the taxonomic structure and the display of all the values in which this characteristic may be expressed in a given domain, addressing the issue of under which different aspects the characteristic may be expressed. To describe this process, Ranganathan uses expressions as "unitary group," "unitary class," and "individualization" (Ranganathan 1967, 57-59).

Ranganathan proposes an analysis process which exhausts the possibilities of values to that characteristic in a domain, a process he names "Complete assortment of a Universe" by the application of a "Scheme of assortment" (Ranganathan 1967, 58). Examples include a classification schema to boys and their characteristics. Answering such a question, the Theory of Faceted Classification enables the generation/classification of a specific expression of a compound subject (by concatenating the values of different facets) in the classification device and at the same time, to define an access point to further information recovery.

The importance of the facet approach is that it stresses that several characteristics may be considered simultaneously in the development of taxonomic structures for a domain thus resulting in several aspects or hierarchies by which knowledge records may be accessed. This is due to the fact that faceted analysis aims not one canonical taxonomy but a retrieval device to attend to the variety of users needs: "different descriptions are correct: each of them expresses a facet of the object. Yet they are all descriptions of the same object. Hence, one of the main tasks of information science is to find ways to integrate different descriptions of the same object" (Gnoli and Poli 2004, 152).

Actually, faceted analysis is useless if it is limited to just one facet, thus resulting in just a single hierarchy, not different from the hierarchical enumerative classification systems. The usefulness of facet analysis as a retrieval mechanism is that several aspects can be revealed thus resulting in multiple access points to a knowledge records collection. In addition to each facet being derived from a class, faceted analysis also proposes principles for the preferred order to present the values in each facet, i.e., the concepts in an array.

\subsection{Discussion}

Traditional classification systems have always being concerned with a disciplinary approach. The pitfalls of such an approach are beginning to be questioned. Gnoli (2008, 178) writes:

Many scholars in bibliographic classification have observed that the disciplinary approach is not the only possible one, and that together with benefits (like reflecting the most frequent approach of researchers) it also brings limitations, especially for interdisciplinary and innovative research. Indeed, disciplines act as a canonical grid.

This questioning leads to the examination of other approaches as feasible for developing a KOS (Gnoli and Poli 2004, 157): "Such a possibility of shifting from disciplines to phenomena as the base unit for the structure of classification has been remarked in recent decades by several researchers in classification."

Ontology comes into play as a viable strategy with which, for example, to construct robust domain models. An ontological grounded knowledge of the objects of the domain should make their codification simpler, mode transparent, and natural. Indeed, ontology can give greater robustness to models by furnishing criteria and categories by which to organize and construct them (Gnoli and Poli 2004). Gnoli (2009, 2) stresses the need that a KOS should have a solid ontological basis: "it is believed that as more accurately is reality reflected in a KOS, as more effective will it be even for practical applications."

On the other hand, computer science has long faced the problem of representing domains in a digital environment. That means achieving accuracy, formality, logical and unambiguous semantics in developing knowledge representation schemes in the absence of human assistance. Since the 1970s, the computer industry realized the increasing cost of developing computer systems which do not correspond to users needs. The faults of computer system developers in clearly understanding user's needs concerning the system let to the development of methodologies aimed at accurately capturing the systems' requirements. An essential requirement of such methodologies was that they may be a communication tool between system developers and users. Mylopoulos (1992, 20) defines conceptual modeling as "the activity of formally describing the physical and social world around us for the purpose of understanding and communication." 
Pioneers in the development of such methodologies were Ed Yourdon and Larry Constantine (1975), Chris Gane and Trish Sarson (1977), Tom de Marco (1979), and others. These methodologies, also called structured analysis or system engineering, clearly divide the system development process into two distinct phases: requirement analysis, which produces a logical model of the system to be developed, and software implementation, which concerns the development of programs, user interface design, testing, tuning, and installation of the application in a computer production environment.

While conceptual methodologies as structured analysis has its focus on processes, another pioneer, Peter Chen (1976) proposed the Entity-Relationship (E-R) model, a methodology which has a focus on entity, represented as an aggregate of attributes, i.e., data, and their relations with other entities. The E-R methodology is aimed at designing databases. Since then, conceptual modelling has been an important focus of research in computer science. The primary product of such methodologies is what was called the conceptual model, usually a graphical diagram.

Researchers as Guarino and Guizzardi (2006), Guizzardi (2005), and many others have emphasized the need that conceptual modeling should have solid ontological bases. Nowadays, many meetings and workshops focus on the convergence of ontology, conceptual modeling, and software engineering. Computer science considers conceptual modeling, including increasingly ontological analysis (Guizzardi 2005), as an essential phase of system development. There is also an increased use of conceptual models in KO, such as the FRBR model (IFLA 1998) and the CIDOC-CRM, as guides to the development of a KOS in digital environments. The development of a KOS in digital environments is a motivating factor to the adoption of conceptual modeling, incorporating the advances on ontological analysis and the formalism provided by computer ontologies (Giunchiglia et al. 2009) in the current KOS development.

The role of faceted classification as bases for a KOS on the Web has been emphasized by many authors including Denton (2009), Gnoli and Hong (2006), Priss (2008), Putkey (2011), Uddin (2007), and Vickery (2008a). This fact points toward a rapprochement between $\mathrm{KO}$ and conceptual modelling and the need to integrate faceted analysis within conceptual modelling methodologies (Prieto-Díaz 2003). A proposal in this direction is Poli and Obrst (2010), who suggest a framework for domain modelling comprising foundational ontologies, crossdomain ontologies, domain specific ontologies, and faceted ontologies within a domain, reflecting the various aspects of interest in a domain.

Once the basic ontological structure of a domain has been established - that is to say, once the levels of reality of the domain have been fixed-the subsequent step is to devise their dimensions of analysis. Here is where faceted analysis can best play its role. Maintaining our reference domain of biology, two series of facets follow. The first series is centered on the governing concept of organism as an individual whole and lists the "viewpoints" from which organisms so taken can be seen. (Poli and Obrst 2010, 15).

The second series of facets list all the other viewpoints, those not focused on the organism as a whole. These may comprise, for instance, genetics (focus on the genes), ethology (focus on some population of organisms), and ecology (focus on an entire ecosystem). But, again, this is not the entire story. A substantial number of other facets can and should be developed, concerning, for instance, the growth and development of organisms, their reproduction, or their alimentation. For each of these facets, appropriate ontologies can be developed. (Poli and Obrst 2010).

This position suggests that faceted analysis may be a modeling phase to be developed after ontological analysis and the definition of the domain core categories comprising the domain ontology. As a methodological phase, faceted analysis may thus indicate possible access points and issues related to the interface design phase.

\subsection{Concluding remarks}

Formal ontology aims at defining what exists, here and now, and it looks for ontological foundations of what exists. Moreover computational ontology and $\mathrm{KO}$ both develop methodologies to model specific domains; domain modeling is a basic, common activity to both disciplines. However the faceted analysis phase has a pragmatic approach to domain modeling, aiming at developing an efficient KOS to providing access to knowledge records.

$\mathrm{KO}$ cannot ignore the knowledge provided by ontology, as it reveals the ultimate nature of what does exists. If knowledge domains were not represented in an ontologically consistent way in digital environments, as stressed by Gnoli, computational inferences based on them will lead to inconsistencies. As knowledge is produced and recorded according to its nature, the properties and different aspects of how things exist are viewed or thought by users; so it may be accessed and organized accordingly. Ontology provides the methodological tools for modeling domains in an ontologically consistent way.

In conclusion, $\mathrm{KO}$ - faceted analysis-aims at identifying all possible aspects of a phenomenon which may be of interest in order to preview users' information needs. It always works with users' needs related to different aspects of a domain. The needs of contemporary culture imposes to $\mathrm{KO}$ that it must now develop KOS in digital 
environments. Limitations of computers' capacities for logical and semantic processing imposes the requisite of developing computational representations that are logically and ontologically coherent Those requisites are much more rigid in the present digital environment than when old KOS were operated by information professionals and several assumptions were implicit based on empirical experience of their operators.

Furthermore, $\mathrm{KO}$, in face of the exponential growth of knowledge that characterizes contemporary society should record it in formats that increasingly enable semantic processing and inferences by programs. Some initiatives towards this direction are the FRBR Core vocabulary/namespace coded in RDF and identified by a URI (Newman and Davis 2005), and SKOS (2004), a model for describing KOS as thesauri and taxonomies, both to be used in semantic Semantic Web applications. From a wide perspective, $\mathrm{KO}$ is user oriented, takes into account the users' mental models and needs in seeking information to make sense of their praxis, and has, as the objective, the development of information access and recovery systems aimed at answering the question of how to search for information and how be informed.

\section{References}

Berners-Lee, Tim, Hendler, James and Lassila, Ora. 2001. The semantic web. Scientific American. Available http:// www.scian.com/2001/0501 issue/0501berners-lee.html.

Buckland, Michael. 1991. Information as thing. Journal of the American Society of Information Science. 42: 351-60. Available http://www.sims.berkley.edu/ buckland/ thing.html.

Chen, Peter Pin-Shan. 1976. The entity-relationship model-Toward a unified view of data. ACM transactions on database systems 1: 9-36.

CIDOC-CRM. 2013. The CIDOC conceptual reference model. Available http://www.cidoc-crm.org/

Cutter, Charles A. 1904. Rules for a Dictionary Catalog. Washington: Government Printing Office.

Davis, Randal, Shrobe, Howard and Szolovits, Peter. 1993. What is a knowledge representation? AI Magazine 14: 17-33. Available http://groups.csail.mit.edu/ medg/ftp/psz/k-rep.htm.

Denton, Willian. 2009. How to make a faceted classification and put it on the web. [Toronto]: Miskatonic University Press. Available http://www.miskatonic.org/library/ facet-web-howto.html.

IFLA Study Group on the Functional Requirements for Bibliographic Records. 1998. FRBR - Functional Requirements for Bibliographic Records: final report. 1998. Munchen: K.G. Saur.
Foskett, Antony Charles. 1996. The subject approach to information. London: Library Association Publishing.

Gane, Chris and Sarson, Trish. 1977. Structured system analysis: Tools and techniques. [Texas]: McDonnell Douglas Systems Integration Company.

Giunchiglia, Fausto, Dutta, Biswanath and Maltese, Vincenzo. 2009. Faceted lightweight ontologies. In Conceptual modeling foundations and applications, lecture notes in computer science 5600: 36-51. Available http://link. springer.com/search?facet-author=

Gnoli, Claudio. 2008. Categories and Facets in Integrative Levels. Axiomathes 8: 177-92.

Gnoli, Claudio. 2009. The ontological approach to knowledge organization. Invited paper sent for discussion at the 2 nd Seminar on Ontology Research, Rio de Janeiro, 21-22 September 2009.

Gnoli, Claudio and Mei, Hong. 2006. Freely faceted classification for web-based information retrieval. New review of bypermedia and multimedia 12: 63-81. Available http://dx.doi.org/10.1080/13614560600758944.

Gnoli, Claudio and Poli, Roberto. 2004. Levels of reality and levels of representation. Knowledge organization 31: 151-60.

Guarino, Nicola. 1995. Formal ontology, conceptual analysis and knowledge representation. International Journal of Human Computer 43: 625-40. Available http://nemo.nic. uoregon.edu/wiki/images/7/79/Guarino_IJHCS1995_ Formal_Onto_conceptual_analysis.pdf.

Guarino, Nicola. 1997. Some organizing principles for a unified top-level ontology. Padova: National Research Council.

Guarino, Nicola. 1998. Formal ontology in information systems. In Guarino, Nicola, ed., Proceedings of FOIS'98, Trento, Italy, 6-8 June 1998. Amsterdam: IOS Press, pp. 315.

Guarino, Nicola and Guizzardi, Giancarlo. 2006. In the defense of ontological foundations for conceptual modelling. Scandinavian journal of information systems 18 : 115-26.

Guarino, Nicola and Welty, Christopher A. 2009. An overview of OntoClean. In: International handbook on information systems. [Berlin]: Springer, pp. 201-20. Available http://wiki.loa-cnr.it/Papers/GuarinoWeltyOnto Cleanv3.pdf.

Guizzardi, Giancarlo. 2005. Ontological foundations for structural conceptual models. Enschede, The Nederlands: CTIT, Telematica Institut.

Hjørland, Birger. Semantics and knowledge organization. 2007. Annual review of information science and technology 41: 367-406.

Hjørland, Birger and Albrechtsen, Hanne. 1995. Toward a new horizon in information science: Domain analysis. Journal of the American Society for Information Science 46: 400-25. 
De Marco, Tom. 1979. Structured Analysis and system specification. Englewood Cliffs, N.J.: Prentice Hall.

Masolo, Claudio, Vieu, Laure, Bottazzi, Emanuele, Catenacci, Carola, Ferrario, Roberta, Gangemi, Aldo and Guarino, Nicola. 2004. Social roles and their descriptions. In Dubois, Didier, Welty, Christopher and Williams, Mary-Anne, eds., Proceedings of $9^{\text {th }}$. International Conference on the Principles of Knowledge Representation and Reasoning (KR, 2004), Whistler, Canada, June 2-5, 2004, [Palo Alto, California]: AAAI Press, pp. 267-77.

McGuinness, Deborah L. 2003. Ontologies come to age. In Fensel, Dieter, Hendler, Jim, Lieberman, Henry, and Wahlster, Wolfgang, eds., Spinning the semantic web: Bringing the World Wide Web to its full potential. Massachusetts: MIT Press. Available http://www.ksl.stanford. $\mathrm{edu} /$ people/dlm/papers/ontologies-come-of-age-mitpress-(with-citation).htm.

Le Moigne, Jean-Luis. 1990. A teoria do sistema geral. Lisboa: Instituto Piaget.

Mylopoulos, John. 1992. Conceptual modelling and Telos. In Loucopoulos, Pericles and Zicari, Roberto, eds., Conceptual modelling, databases, and CASE: An integrated view of information systems development. New York: Wiley, pp. 49-68.

Newman, Richard and Davis, Ian. 2005. Expression of core FRBR concepts in RDF. Available http://vocab. org/frbr/core.html.

Poli, Roberto and Obrst, Leo. 2010. The interplay between ontology as categorial analysis and ontology as technology. In: Theory and applications of ontology: Computer applications. [Berlin]: Springer, pp.1-26.

Prieto-Diaz, Rubén. 2003. A faceted approach to building ontologies. In IRI 2003. IEEE International Conference on Information Reuse and Integration, 2003, pp. 458-65.

Priss, Uta. 2008. Facet-like structures in computer science. Axiomathes 18: 243-55, DOI 10.1007/s10516007-9023-y.

Putkey, Theresa. 2011. Using SKOS to express faceted classification on the semantic web. Library philosophy and practice. Available http://www.webpages.uidaho. edu/ $\sim$ mbolin/putkey.htm.

Ranganathan, Shiyali Ramamrita. 1967. Prolegomena to a library classification. Bombay: Asia Publishing House.
Ranganathan, Shiyali Ramamrita. 1968. Choice of a scheme for classification. Available http://www.isibang.ac.in/ $\sim$ library/portal/Pages/chp5.pdf.

Saracevic, Tefko. 1995. A natureza interdisciplinar da ciência da informação. Ciência da Informação 24 n1. Available http://revista.ibict.br/ciinf/index.php/ciinf/article/ view $/ 530 / 482$

Saracevic, Tefko. 1975. RELEVANCE: a review of and a framework for thinking the notion in Information Science. Journal of the American Society for Information Science 26: 321-43.

SKOS. 2004. Simple knowledge organization system. 2004. Available http://www.w3.org/2004/02/skos/.

Smith Barry. Ontology and information systems. 2006. Available http://ontology.buffalo.edu/ontology $\% 28 \mathrm{PIC} \%$ 29.pdf.

Smith, Barry. and Grenon, Pierre. 2004. The Cornucopia of formal-ontological relations. Dialectica 58: 279-96.

Smith, Barry and Welty, Christopher. 2001. Ontology: Towards a new synthesis. In Welty, Christopher and Smith, Barry, eds., Formal ontology in information systems. Ongunquit, Maine: ACM Press. Available portal.acm. org $/$ citation.cfm?doid=505168.505201.

Spiteri. Louise. 1998. A simplified model of facet analysis. Canadian journal of information and library science 23: 130. Available http://www.iainstitute.org/en/learn/ research/a_simplified_model_for_facet_analysis.php.

Uddin, Mohammad Nasir and Janecek, Paul. 2007. Faceted classification in web information architecture: a framework for using semantic web tools. The electronic library $25 \mathrm{n} 2: 15$.

Vickery, Brian. 2008a. Faceted classification for the web. Axiomathes 18: 145-60, DOI 10.1007/s10516-0079025-9.

Vickery. Brian. 2008b. On knowledge organization. 2008b. Available http://classic-web.archive.org/web/2008040 4103206/www.lucis.me.uk/knowlorg.htm.

Wersig, Gernot and Neveling, Ulrich. 1975. The phenomena of interest to information science. The information scientist 9: 127-40.

Yourdon, Edward and Constantine, Larry L. 1975. Structured design. New York: Yourdon Press. 